\title{
Gestão em saúde na pandemia do COVID-19
}

\author{
COVID-19 pandemic health management
}

Renata Garcia Duarte'; Jacqueline Garcia Duarte²

1. Centro Universitário Newton Paiva, Belo Horizonte, Minas Gerais, Brasil
2. Universidade Federal de Minas Gerais, Belo Horizonte, Minas Gerais, Brasil

\section{Resumo}

Introdução: O aparecimento do Coronavírus da síndrome respiratória aguda grave 2 (SARS-CoV-2), em dezembro de 2019, evidenciou a potencialidade de dispersão rápida e mundial do vírus. Em pouco tempo, uma parcela substancial da população global foi acometida pela doença do Coronavírus (COVID-19) e a rápida evolução grave da doença evidenciou a demanda urgente por mudanças na gestão de saúde. Objetivo - Compilar os novos parâmetros de gestão em saúde aplicados à pandemia. Metodologia - Foi feita uma revisão narrativa, que compilou artigos e informações obtidas no ano de 2020, em âmbito nacional e internacional. Resultados - De uma forma geral, foi observada uma iniciativa político-econômica para direcionar verbas ao combate e ao controle do vírus. Em âmbito social, foram traçados protocolos de prevenção da dispersão do vírus a serem seguidos por toda a população. Nas instituições de saúde, a gestão focou na alteração das características das unidades e na administração: do quadro de funcionários; da carga horária; do fluxo de deslocamento; do estoque e fornecimento de equipamentos de proteção individual (EPI) e de testes diagnósticos; da saúde mental da equipe. A Telessaúde utilizou da tecnologia para manter a continuidade da assistência para pessoas. Mas apesar dos esforços, ainda existem deficiências no processo a serem sanadas. Conclusão - A urgência da pandemia de COVID-19 deixa claro que, apesar da rápida adaptação da gestão em saúde, muitas limitações do processo existiram. Assim, há uma necessidade por um planejamento mais cauteloso, complexo e preventivo para futuras calamidades.

Palavras-chave: Gestão em saúde; COVID-19; SARS-CoV-2; Gestão Hospitalar; Telessaúde. 


\begin{abstract}
Introduction: The emergence of severe acute respiratory syndrome coronavirus 2 (SARSCoV-2) in December 2019 showed the capability of fast and global spread of the virus. In a short time, a substantial portion of the global population was affected by the Coronavirus disease (COVID-19) and the rapid severe evolution of the disease showed the urgent demand for change in health management. Objective - To compile the new health management parameters applied to the pandemic. Methodology - A narrative review was carried out, which compiled articles and information obtained in the year 2020 at national and international levels. Results - In general, a political-economic initiative was observed to direct funds to combat and control the virus. In the social sphere, protocols for preventing the spread of the virus were designed to be followed by the entire population. In health institutions, management focused on changing the characteristics of the units and administration: the staff; the workload; displacement flow; the stock and supply of personal protective equipment (PPE) and diagnostic tests; mental health of the team. But despite efforts, there are still deficiencies in the process to be remedied. Conclusion - The urgency of the COVID-19 pandemic makes it clear that despite the rapid adaptation of health management, many limitations of the process existed. Thus, there is a need for a more careful planning, complex and preventive for future calamities.
\end{abstract}

Keyword: Health management; COVID-19; SARS-CoV-2; Hospital management; Telehealth.

Recebido em: 08-12-2020 Publicado em:29-10-2020

\title{
Autor correspondente
}

Jacqueline Garcia Duarte

Avenida Presidente Antônio Carlos, 6627

Pampulha - 31270-901, Belo Horizonte, MG - Brasil

jacquegbiologia@gmail.com

\section{Introdução}

A doença do Coronavírus (COVID-19) é a determinação do quadro clínico apresentado por pacientes acometidos pelo beta coronavírus conhecido como Coronavírus da síndrome respiratória aguda grave 2 (SARS-CoV-2)'. Esse microrganismo é um vírus de fita simples de RNA da subfamília Orthocoronavirinae, da família Coronavirinae, e da ordem Nidovirales. Ele foi encontrado, inicialmente, em dezembro de 2019, na cidade de Wuhan, província de Hubei, China². Em 30 de janeiro, a Organização Mundial da Saúde (OMS) declarou a COVID-19 uma emergência de saúde pública de interesse internacional e, logo em seguida, classificou-a como uma pandemia, em 11 de março de $2020^{3,4}$. A pandemia dispersou rapidamente pelo mundo todo tornando um desafio robusto para governos, indivíduos, sociedade como um todo e a comunidade científica ${ }^{5,6}$.

Mas o coronavírus não surgiu no mundo em 2019. Na verdade, esta é descrita como a terceira ocorrência de transmissão 
dessa família de vírus. O primeiro surto ocorreu em 2002, em Guangdong, na China pelo vírus da Síndrome respiratória aguda grave (SARS-CoV). Em 2012, houve um novo surto em Jeddah, na Arábia Saudita, pelo vírus da Síndrome respiratória do Oriente Médio (MERSCoV) ${ }^{7}$.

As principais formas de transmissão dessa família de vírus se dão por dispersão de gotículas, disparadas no momento da fala, espirro, tosse e por contato. Acreditase que as gotículas respiratórias sejam a via predominante na forma da transmissão desse vírus. A transmissão por contato se dá mediante à interação do indivíduo com superfícies contaminadas ou com fluidos corporais contendo o vírus, presentes na cavidade oral, cavidade nasal e outras membranas mucosas 8,9 .

A população, no geral, é suscetível, mas os mais velhos foram responsáveis por alavancar os casos de COVID-19 seguidos de adultos, jovens e crianças. Os pacientes com comorbidades incluindo hipertensão, diabetes, infecção respiratória pré-existente, doença cardiovascular e câncer têm maior chance de desenvolverem as formas mais graves de COVID-19 e apresentam maior risco de desenvolver complicações ${ }^{8}$. Os profissionais e os trabalhadores de saúde têm altos riscos de infecção por estarem em contato frequente com pacientes infectados ${ }^{10}$.

O período médio de incubação da COVID19 é de 5,1 dias, havendo casos que desenvolveram sintomas em até 11,5 dias, e pode afetar qualquer indivíduo independentemente da idade e do sexo ${ }^{8,11}$. Esse período corresponde à presença do vírus no organismo, com dispersão, mas sem presença de sintomas pelo infectado. Os sintomas leves a moderados afetam a maioria dos pacientes ${ }^{12}$. Além disso, há pacientes que não apresentam nenhum sintoma durante todo o processo infeccioso, são os chamados assintomáticos, que também estão propícios a transmitir a COVID-196.

Os sintomas iniciais são inespecíficos caracterizados por febre, tosse seca e falta de $a r^{8,13}$. Pacientes mais graves apresentam dor faríngea, dispneia, tontura, dor abdominal e anorexia, além do quadro inflamatório pulmonar característico. Pacientes que desenvolveram ao óbito apresentaram diversos quadros clínicos, dentre eles, linfopenia grave, choque, síndrome da dificuldade respiratória aguda (SDRA), arritmia e lesão cardíaca aguda ${ }^{14}$.

Até a última consulta do relatório internacional, realizada no dia 08 de dezembro de 2020, a OMS havia informado que, no mundo, havia um total de 66.729 .375 casos confirmados e 1.535.982 mortes. No Brasil, eram 6.603 .540 casos confirmados e 176.941 mortes ${ }^{5}$. Números esses que ainda não param de subir.

A dispersão global tão rápida desse novo vírus com o acometimento, de desenvolvimento moderado a grave, por uma parcela considerável de seus pacientes, configurou um grande desafio para os gestores, médicos e demais profissionais da saúde. Houve uma necessidade de mudança de parâmetros gestacionais das unidades hospitalares.

Mediante a todos esses fatos, o presente trabalho tem como objetivo compilar os novos parâmetros de gestão em saúde aplicada à pandemia de COVID-19, para melhor atender a demanda crescente dos casos.

\section{Metodologia}




\section{Desenho e configuração do estudo}

A revisão narrativa abrange toda a bibliografia já tornada pública em relação a um determinado tema, incluindo publicações avulsas como boletins, jornais, revistas, livros, pesquisas, monografias, teses entre outros ${ }^{15}$. Diante do assunto levantado para a construção da presente revisão narrativa, foi estabelecida a seguinte questão norteadora: Quais são as estratégias utilizadas pelos gestores, médicos e demais profissionais da saúde para o enfrentamento da COVID-19?

A presente revisão foi estruturada da seguinte forma: Determinação do assunto específico centrado no objetivo da revisão; busca dos artigos nas bases de dados Sistema Online de Busca e Análise de Literatura Médica (MEDLINE), Literatura Latino-Americana e do Caribe em Ciências da Saúde (LILACS) e Biblioteca Nacional de Medicina dos Estados Unidos (PUBMED) e Google Acadêmico; seleção dos artigos por meio da leitura dos títulos e resumos; leitura dos trabalhos na íntegra e coleta de dados. Como forma de complementar a revisão narrativa, também foram consultados os sites: OMS, Organização Pan-Americana da Saúde (OPAS) e Fundação Oswaldo Cruz (FIOCRUZ).

Foram considerados artigos em português e inglês, por se tratar de uma pandemia mundial que retrata a situação global, provocada pela contaminação da COVID-19. Os artigos sobre o tema foram publicados no ano de 2020. Objetivou-se relatar a situação global perante COVID19, as mudanças quanto à gestão em saúde, junto aos desafios dos profissionais na rápida resposta de combate ao vírus. Foram desconsiderados artigos que não condiziam com o assunto.
O acesso às bases de dados facilitou a busca dos termos, que foram "COVID-19", "gestão em saúde na pandemia de COVID-19", "gestão hospitalar na pandemia de COVID-19", "gestão enfermagem na pandemia de COVID-19", "telessaúde COVID-19", "teleconsulta COVID-19", "health management in the COVID-19 pandemic", "hospital management in the COVID-19 pandemic", "nursing management in the COVID-19 pandemic", "COVID-19 telehealth", "COVID-19 teleconsultation".

A análise e resultados obtidos foram feitos de forma descritiva, possibilitando ao leitor uma vasta amostra do que a COVID19 pode causar e do quanto é importante valorizar os profissionais da saúde e a prevenção.

\section{Resultados e Discussão}

Gestão em Saúde no contexto da COVID19

A gestão nada mais é que a capacidade em dirigir, administrar. $\bigcirc$ modelo atual apresenta uma centralização do poder nos gestores para melhor enfrentar questões e desafios, mas permite certa autonomia e iniciativa dos funcionários em prol de uma melhor qualidade de serviço e aumento da produtividade. A gestão em saúde é classificada como uma atenção gerenciada, que visa aumentar a eficácia do trabalho em saúde na medida em que controla e determina 0 cotidiano dos trabalhadores ${ }^{16}$.

A atual pandemia evidenciou a importância da gestão em saúde na aplicação de medidas de contenção e tratamento da COVID-19. Em um curto 
prazo, medidas de contingência, controle e proteção precisaram ser tomadas visando ao recuo dos índices de infecção e à manutenção da saúde da população e dos profissionais de saúde em combate ${ }^{17-}$ 20. A gestão em saúde aborda aspectos relacionados com o planejamento, programação, reorganização do processo de trabalho, sistemas de informação em saúde, capacitação de pessoal, práticas de monitoramento, supervisão e avaliação de sistemas e serviços de saúde. Além de, também, abranger a área de informação gerencial e comunicação em saúde absorvendo as evoluções tecnológicos no auxílio do gerenciamento de dados de saúde ${ }^{21}$.

Investimentos governamentais no combate à pandemia da COVID-19

Após os governos identificarem a COVID19 como uma doença infecciosa, foram adotadas medidas preventivas e de contenção. Iniciativas econômicas de investimento governamental foram necessárias para o combate ao vírus. No Brasil, com a Lei Complementar $n^{\circ} 172$, de 15 de abril de 2020, foram liberados recursos parados nos fundos de saúde de estados e municípios para ações de combate à pandemia. A princípio, estimou-se o aumento em R $\$ 6$ bilhões para essa finalidade 22 .

De semelhante modo, foi realizado em outros países, a exemplo, nos Estados Unidos da América, por meio da lei de Auxílio, Socorro e Segurança Econômica (CARES) do Coronavírus, em 27 de março de 2020, com o objetivo de fornecer aproximadamente US\$ 2 trilhões em ajuda e assistência a indivíduos, empresas, governos estaduais, prestadores de serviços de saúde e outros afetados pelo surto do COVID-1923.
Apesar dos valores de investimento entre os países apresentarem uma discrepância imensa, é importante salientar que cada um apresenta uma realidade econômica. Soma-se a isso o fato de possuírem outras características diferentes, como extensão de território e número total da população.

Adaptações das instituições de saúde na pandemia da COVID-19

De uma forma generalizada, as instituições de saúde pública e privada apresentaram mudanças amplas para enfrentar o vírus. Dentre elas, houve a criação de um comitê de crise; realização de treinamento e preparo da equipe; cancelamento de cirurgias eletivas; reforço e distribuição de equipamentos de proteção individual (EPI); criação de fluxos para a diminuição de tráfego de pacientes suspeitos; alteração nas características das unidades; aumento no número de leitos destinados à COVID-19; gestão de testes diagnósticos; contratação de pessoal e apoio psicológico a equipe $17-20,23,24$.

O comitê de crise, entre outros componentes, apresenta representantes médicos e enfermeiros. As reuniões ocorrem diariamente e buscam estudar estratégias para o melhor atendimento ${ }^{18,19}$. Faz-se relevante, com essa informação, salientar a importância do profissional enfermeiro. Muitas vezes esquecido e desvalorizado, este profissional ocupa local de destaque no enfrentamento da pandemia ${ }^{25}$. Os enfermeiros são os profissionais da saúde que mais correm risco de se infectar com a COVID-19. Isso se deve ao fato de serem os profissionais que mais tempo ficam em contato com os pacientes, desenvolvendo cuidados e liderando políticas de 
humanização ${ }^{17}$. Muito além de atuarem diretamente na assistência, eles também colaboram com o planejamento e funcionamento da estrutura física, a gestão de recursos humanos e a construção de protocolos e fluxos de cuidado 26 .

Os treinamentos de preparo da equipe visam, ainda que parcial ou informalmente, capacitar as equipes. Neles são abordados temas, como a microbiologia do vírus, forma de contágio, sinais e sintomas; uso correto de EPI e fluxo de atendimento ${ }^{18,19}$. Orientações, como a lavagem adequada das mãos, uso do álcool líquido e em gel a 70\% e evitar o toque na face, olhos, boca e nariz mantem-se regularmente ${ }^{19}$.

- cancelamento de cirurgias eletivas busca disponibilizar o ambulatório cirúrgico para o acolhimento de pacientes suspeitos. Mais além, visa reduzir o risco de contágio e transmissão entre pacientes ${ }^{18}$.

A distribuição de EPIs demandou uma incrementação e um política de uso racional, uma vez que é uma precaução necessária para impedir o contágio. São disponibilizadas ao profissional de saúde máscaras simples, máscaras N95, face shield, capote, aventais e óculos ${ }^{18,19}$.

A criação de fluxos procurou restringir a circulação do vírus. Salienta-se a importância do ambulatório para triar os pacientes suspeitos, restringindo seus locais de acesso. Nas Unidades de Pronto Atendimento (UPAs), destaca-se a criação do Centro Ambulatorial de Triagem (CAT). $\bigcirc$ atendimento ambulatorial visa separar os pacientes por meio da classificação de risco e priorizar o atendimento daqueles que apresentam sintomas respiratórios, bem como isolar os pacientes graves ${ }^{18,19,26}$.

A alta procura de pacientes com sintomas respiratórios, também, demandou alteração nas características das unidades. Nesse sentido, a desocupação dos leitos de internação na Clínica Médica, Ortopedia e Ginecologia e a relocação para pacientes com suspeita foram necessárias. $\bigcirc$ aumento da disponibilidade no número de leitos nas Unidade de Cuidados Intensivos (UTIS) seguiu a mesma lógica, dando prioridade a esses pacientes, bem como inaugurando novos leitos ${ }^{18,19,24,26}$.

A gestão dos testes diagnósticos viabilizou o acompanhamento dos pacientes visando responder rapidamente aos sinais. Prioriza-se o uso de exames como o da proteína C-reativa (PCR), a partir de amostra de esfregaço da rinofaringe e o de sorologia SARS-COV-2, a partir do sangue. O monitoramento virológico se faz necessário até que haja a negativação do exame $P C R^{20}$.

A ininterrupta demanda por cuidados demandou a contratação de mais profissionais. Também permitiu fortalecer a equipe com profissionais com experiência em cuidados críticos, visando à readequação do dimensionamento conforme a complexidade do serviço ${ }^{18,26}$.

O apoio psicológico para a equipe também se tornou uma estratégia imprescindível para o enfrentamento da pandemia. Relatos de aumento dos sintomas de ansiedade, depressão, perda da qualidade do sono, aumento do uso de drogas e medo de se infectarem ou transmitirem a infecção aos membros da família são recorrentes. Estes profissionais estão expostos a muita pressão. Eles correm risco real de se infectarem, muitas vezes estão em uso de proteção inadequada, apresentam excesso de trabalho, frustração, discriminação, precisam fazer isolamento, têm o contato reduzido ou até não têm contato com a família. Todos esses fatores somados afetam a saúde mental e comprometem 
o entendimento e a capacidade de tomada de decisões desses profissionais ${ }^{10}$. Assim, a presença de um psicólogo no próprio local de trabalho se fez necessária, com atendimentos individualizados e em grupo, durante as reuniões de equipe $19,26,27$.

Prevenção e controle da disseminação da COVID-19

Outro ponto muito importante da gestão em saúde no combate à disseminação do vírus, transcende os muros das instituições de saúde, e atinge a população geral. Ele consiste na conscientização e na disseminação de protocolos de prevenção. Nesse sentido, a população é constantemente alertada ao uso de máscaras em locais públicos, lavagem frequentes das mãos, uso de álcool e a manutenção do distanciamento social ${ }^{6}$. As autoridades atuam como auxiliares no processo, ao conter a movimentação e a aglomeração de pessoas ${ }^{28}$.

Comunicação Digital na Pandemia da COVID-19

Um ponto de destaque da gestão em saúde que se fortaleceu na pandemia foi a assistência digital à saúde. A Telessaúde, de acordo com o Ministério da Saúde brasileiro, é o "uso de tecnologias da informação e comunicação para atividades à distância relacionadas à melhoria da saúde da população por meio da qualificação do SUS". De acordo com a Portaria GM/MS n².546/2011, os Núcleos de Telessaúde podem desenvolver atividades técnico-científicas e administrativas para o planejamento, execução, monitoramento e avaliação das seguintes vertentes: teleconsultoria, telediagnóstico, tele-educação, segunda opinião formativa, apoio na perspectiva da educação permanente para qualificar as Equipes de Atenção Básica e ampliação da resolutividade ${ }^{29}$.

A Telessaúde, ao garantir o atendimento a distância, reduz a transmissão viral, limita o contato entre as pessoas e, ao mesmo tempo, permite que as pessoas infectadas por COVID-19 com desenvolvimento leve e pessoas com doenças não transmissíveis (DNTs) sejam tratadas em suas residências. Dessa forma, sua função primária é manter a continuidade da assistência para pessoas durante a pandemia ${ }^{30,31}$.

Como mencionado no informativo publicado em 11 de agosto de 2020 pela OPAS, a Telessaúde pode ser utilizada pelo paciente para autocontrolar suas doenças junto ao médico, mas também por profissionais da saúde e cuidadores que precisam de auxílio no cuidado de um paciente. Dentre as diversas aplicações da Telessaúde, ela pode ser usada no controle e triagem de pacientes durante a pandemia; no monitoramento à distância dos casos em tratamento de COVID-19; no acompanhamento após alta; na busca rápida de opinião de outros profissionais para casos graves; na troca de experiências nacionais e internacionais; no acesso a serviços de telerradiologia; e nos treinamentos online para profissionais de saúde ${ }^{30,31}$.

No âmbito mundial, muitos países vêm desenvolvendo aplicativos que rastreiam pessoas e o progresso da doença, além de disseminar informações para conscientização sobre os cuidados. Alguns exemplos são TraceTogether (Singapura), CovidWatch (EUA), PeduliLindungi (Indonésia), Kwarantana Dommowa (Polônia), HaMagen (Israel), 
Aarogya Setu (Índia). No Brasil, há o aplicativo Coronavírus - SUS, criado pelo Ministério da Saúde e disponível para download gratuitamente ${ }^{32}$.

No âmbito nacional, muitas categorias profissionais possuem respaldo legal para fazer uso da Telessaúde. Durante a pandemia, enfermeiros ficam autorizados por intermédio da Resolução COFEN No 634/2020. Pelo mesmo período, médicos também são permitidos por meio da Lei 13.989 de 15 de abril de 2020. Psicólogos possuem o direito a prestar serviços psicológicos por telefone, desde fevereiro de 1995, pela Resolução nº 002. Mas desde 25 de setembro de 2000, com a Resolução $n^{\circ}$ 003, também podem atender por computador. Fonoaudiólogos também têm a permissão de realizar a teleconsulta desde $1^{\circ}$ de março de 2013, pela Resolução $n^{\circ} 427^{30}$.

Limitações na Pandemia da COVID-19

Apesar de todo um traçado estratégico e certo investimento financeiro governamental, a pandemia gerou uma incerteza pela impossibilidade de prever sua duração. Nesse sentido, o gerenciamento de verba destinado ao combate ao COVID-19 se tornou um grande problema ${ }^{18}$.

A falta de conhecimento sobre o novo vírus causou um sentimento de despreparo entre os profissionais da saúde. Mais além, a pandemia carreou diversos problemas de saúde a esses profissionais $^{19}$. Um dos principais e alarmantes problemas é a alta taxa de contaminação dos profissionais da linha de frente de combate. Teixeira e colaboradores (2020) citam em seu estudo o aumento da jornada de trabalho e a inadequada higienização das mãos pelos profissionais como as principais causas do alto índice de infecções. Para eles, as longas jornadas de trabalho, mediante contato direto com pacientes, tornam os enfermeiros estressados e exaustos, o que viabilizaria o risco. Nesse sentido, os autores salientam para a necessidade de estabelecer protocolos hospitalares específicos para reduzir o risco de infecção desses profissionais ${ }^{10}$.

Outra limitação da gestão da pandemia foram os efeitos adversos do uso de EPIs. Apesar de serem aliados ao combate e visarem à proteção de quem os utiliza, esses equipamentos também trazem danos, como o alto índice de acometimento cutâneo. Tal dano pode induzir o profissional a não continuar usando o equipamento de proteção, uma vez que já apresenta ulcerações. $\bigcirc$ ato frequente de higienização das mãos também é um grande vilão para a saúde e a integridade da pele. Esse hábito exacerbado pela pandemia está associado a uma maior incidência de dermatite na pele das mãos ${ }^{10}$.

Outro desafio apontado pela pandemia é a fragilidade do Sistema Único de Saúde (SUS). Como apontado por Gleriano e colaboradores, os problemas estão relacionados com o subfinanciamento, o processo de descentralização e a depreciação da infraestrutura de diferentes níveis de atenção. Somado a isso, ainda há uma defasagem relativa a recursos humanos, relacionada a diferentes formas de contratação, precarização do trabalho e baixo investimento em educação permanente. Assim, o combate à pandemia pelo SUS encontra-se fragilizado pela limitação estrutural e de capital humano ${ }^{33}$.

Por fim, a Telessaúde, apesar de ser uma opção inovadora, também se estabelece como uma estratégia frágil. Suas principais barreiras são a dificuldade de 
acesso e manuseio por parte da população, a segurança digital e seus custos. Alguns pacientes também não se sentem confortáveis e seguros ao interagir virtualmente. Mais além, a modalidade virtual reduz o relacionamento entre $\mathrm{o}$ paciente e $\mathrm{O}$ profissional da saúde. Isso pode gerar confusão ou mal entendimento na transmissão de informações de saúde, o que pode gerar preocupações desmedidas 30,32 .

\section{Conclusão}

A eclosão rápida e descontrolada da COVID-19 demonstrou a fragilidade humana frente à natureza biológica de um microrganismo potencialmente fatal. Mesmo com pouco ou nenhum conhecimento sobre o misterioso novo vírus, sem saber o que esperar e como agir para sua extinção, medidas de intervenção de saúde precisaram ser propostas de maneira urgente e aplicadas prontamente.

Investimentos governamentais de suporte foram demandados. Diversas alterações nas instituições de saúde, como alterações nas características da unidade, no quadro de funcionários, na carga horária, no fluxo de deslocamento, na disponibilização de insumos e de equipamentos, foram necessárias. Atendimentos de saúde remotos também foram uma necessidade. Esforços estes que buscavam, da melhor forma, atender a demanda de cuidado e os anseios da população aflita e amedrontada pelo desconhecido. Ao mesmo tempo, toda a população mundial se viu imersa em uma nova realidade preventiva, baseada em máscaras, lavagem de mãos, álcool em gel e distanciamento.
A aplicação do novo normal deixou claro que, apesar dos esforços contínuos, as limitações ainda existem. A pandemia da COVID-19 acendeu um alerta quanto à demanda por um planejamento mais cauteloso, complexo e preventivo para futuras calamidades.

\section{Declaração de conflito de interesses}

Os autores do artigo declaram que não houve nenhum conflito de interesse que pudesse influenciar o desenvolvimento do trabalho.

\section{Referências}

1. CORONAVIRIDAE STUDY GROUP OF THE INTERNATIONAL COMMITTEE ON TAXONOMY OF VIRUSES. The species severe acute respiratory syndrome-related coronavirus: classifying 2019-nCoV and naming it SARS-CoV-2. Nature Microbiology, v. 5, n. 4, p. 536-544, 2020.

2. ZHU, N.; ZHANG, D.; WANG, W.; LI, X:; YANG, B.; SONG, J.; et al. A Novel Coronavirus from Patients with Pneumonia in China, 2019. The New England Journal of Medicine, v. 382, n. 8, p.727-733, 2020.

3. $\mathrm{WHO}^{\prime}-\mathrm{WHO}$ Director-General's statement on IHR Emergency Committee on Novel Coronavirus (2019-nCoV). In: World Health Organization. Disponivel em: <https://www.who.int/directorgeneral/speeches/detail/who- 
director-general-s-statement-onihr-emergency-committee-onnovel-coronavirus-(2019-ncov)>. Acesso em: 26 out. 2020.

4. $\mathrm{WHO}^{2}-\mathrm{WHO}$ Director-General's opening remarks at the media briefing on COVID-19 - 17 March 2020. In: World Health Organization. Disponível em: <https://www.who.int/dg/speeches/ detail/who-director-general-sopening-remarks-at-the-mediabriefing-on-covid-19---17-march2020>. Acesso em: 26 out. 2020.

5. $\mathrm{WHO}^{3}-\mathrm{WHO}$ Coronavirus Disease (COVID-19) Dashboard. In: World Health Organization. Disponível em: <https://covid19. who.int/> Acesso em: 08 dez. 2020.

6. SHI, Y.; WANG, G.; CAI, X.; DENG, J.; ZHENG, L.; ZHU, H.; et al. An overview of COVID-19. Journal of Zhejiang University-SCIENCE B, v. 21, n.5, p. 343-360, 2020.

7. XIE, M. and CHEN, Q. Insight into 2019 novel coronavirus - An updated interim review and lessons from SARS-COV and MERSCoV. International Journal of Infectious Diseases, v. 94, p. 119-124, 2020.

8. GUAN, W. J.; NI, Z. Y.; HU, Y.; LIANG, W. H.; OU, C. Q.; HE, J. X.; et al. Clinical Characteristics of Coronavirus Disease 2019 in China. The New England Journal of
Medicine, v. 382, n. 18, p.1708-1720, 2020.

9. $\mathrm{WHO}^{4}$ - Coronavirus disease (COVID-19): How is it transmitted? In: World Health Organization. Disponivel em: $<$ https://www.who.int/emergencies /diseases/novel-coronavirus2019/question-and-answers-hub/qa-detail/coronavirus-disease-covid19-how-is-it-transmitted>. Acesso em: 28 out. 2020.

10. TEIXEIRA, C. F. S.; SOARES, C. M.; SOUZA, E. A.; LISBOA, E. S.; PINTO, I. C. M.; ANDRADE, L. R.; ESPIRIDIÃO, M. A. A saúde dos profissionais de saúde no enfrentamento da pandemia de Covid-19, Ciência e Saúde Coletiva, v. 25, n. 9, 2020.

11. LAUER, S.A.; GRANTZ, K.H.; BI Q, JONES, F.K.; ZHENG, Q.; MEREDITH, H.R.; AZMAN, A.S.; REICH, N.G.; LESSLER, J. The Incubation Period of Coronavirus Disease 2019 (COVID-19) From Publicly Reported Confirmed Cases: Estimation and Application. Ann Intern Med. v.172, n. 9, p.577-582.

12. XU, Z.; SHI, L.; WANG, Y.; ZHANG, J.; HUANG, L.; ZHANG, C.; et al.

Pathological findings of COVID-19 associated with acute respiratory distress syndrome. The Lancet. Respiratory Medicine, v. 4, p. 420422, 2020.

13. HUANG, C.; WANG, Y.; LI, X.; REN, L.; ZHAO, J.; HU, Y.; et al. Clinical features of patients infected with 2019 novel coronavirus in Wuhan, 
China. Lancet, v. 395, n. 10223, p. 497-506, 2020.

14. WANG, D.; HU, B.; HU, C.; ZHU, F.; LIU, X.; ZHANG, J.; et al. Clinical Characteristics of 138 Hospitalized Patients With 2019 Novel Coronavirus-Infected Pneumonia in Wuhan, China. JAMA, v. 323, n. 11, p. 1061-1069, 2020.

15. LAKATOS, E. M.; MARCONI, M. A.; Fundamentos de metodologia científica. 7. ed. São Paulo: Atlas, 2010.

16. CAMPOS, G.W. S.; CAMPOS, R.T.O. Gestão em saúde. In: Fundação Oswaldo Cruz. Dicionário da Educação Profissional em Saúde. Disponível em: <http://www.sites.epsjv.fiocruz.br/di cionario/verbetes/gessau.html>. Acesso em: 29 out. 2020.

17. VENTURA-SILVA, J. M. A.; RIBEIRO, O. M. P. L.; SANTOS, M. R.; FARIA, A. C. A.; MONTEIRO, M. A. J.; VANDRESEN, L. Organizational planning in pandemic contexto by COVID-19: implications for nursing management. Journal Heajth NPEPS. V. 5, n.1, 2020.

18. ARAÚJO, P. M. C. G.; BOHOMOL, E.; TEIXEIRA, T. A. B. Nursing management in an accredited public general hospital in the response to the COVID-19 pandemic. Enfermagem em Foco, v.11, n. 1, p. 192-195, 2020.
19. BORDIGNON, J. S.; VARGAS, C. P.; SCHOELLER, S. D.; SANTOS, E. K. A. Experiences and autonomy of nurses in an emergency care unit in pandemic time. Enfermagem em Foco, v.11, n.1, p. 205-210, 2020.

2O. NICASTRI, E.; PETROSILLO, N.; ASCOLI BARTOLI, T.; LEPORE, L.; MONDI, A.; PALMIERI, F.; et al. National Institute for the Infectious Diseases "L. Spallanzani", IRCCS. Recommendations for COVID-19 clinical management. Infectious Disiase Reports, v. 12, n.1, p. 8543, 2020.

21. PAIM, J. S. e TEIXEIRA, C. F. Política, planejamento e gestão em saúde: balanço do estado da arte. Saúde Pública. V. 40, p. 73-78, 2006.

22. GOVERNO FEDERAL BRASILEIRO', 2020 - Lei Complementar $n^{\circ} 172$, de 15 de abril de 2020. In: Diário Oficial da União. Disponível em: <https://www.in.gov.br/en/web/dou /-/lei-complementar-n-172-de-15de-abril-de-2020-252726587>. Acesso em: 27 out. 2020.

23. VACCARO, A. R.; GETZ, C. L.; COHEN, B. E.; COLE, B. J.; DONNALLY, C.J. Practice Management During the COVID-19 Pandemic. Journal of the American Academy of Orthopaedic Surgeons, v. 13, p.10, 2020.

24. GARG, M.; WRAY, C. M. Hospital Medicine Management in the Time of COVID-19: Preparing for a Sprint and a Marathon. Journal of Hospital Medicine, v. 5, p. 305-307, 2020. 
25. MACHADO, M. H.; PEREIRA, E. J.; NETO, X:; GUIMARÃES, F. R.; WERMELINGER, M. C. M. W. Nursing in COVID-19 times in brazil: a look at work management. Enfermagem em Foco, v. 11, n. 1, p. 32-39, 2020.

26. BITENCOURT, J. V. O. V.; MESCHIAL, W. C., FRIZON, G.; BIFFI, P.; SOUZA, J. B.; Maestri, E. Nurse's protagonism in structuring and managing a specific unit for COVID-19. Texto \& Contexto Enfermagem, v. 29, 2020.

27. FUKUTI, P.; UCHÔA, C. L. M.; MAZZOCO, M. F.; CORCHS, F.; KAMITSUJI, C. S.; ROSSI, L.; RIOS, I.C.; et al. How Institutions Can Protect the Mental Health and Psychosocial Well-Being of Their Healthcare Workers in the Current COVID-19 Pandemic. Clinics (Sao Paulo), v. 3, n. 75, p.1963, 2020.

28. NICOLA, M.; O'NEILL, N.; SOHRABI, C.; KHAN, M.; AGHA, M; AGHA, R; Evidence based management guideline for the COVID-19 pandemic - Review article. International Journal of Surgery, $v$. 77, p.206-216, 2020.

29. GOVERNO FEDERAL BRASILEIRO², Obter apoio e qualificação do Telessaúde. In: Governo do Brasil. Rede de Atendimento à Saúde. Disponivel em: $<$ https://www.gov.br/pt- br/servicos/obter-apoio-equalificacao-do-telessaudes. Acesso em: 26 nov. 2020.

30. SACHETT, J. A. G. Adaptation for professional health care in times of COVID-19: contributions from telehealth to the "new normal". Journal Health NPEPS, v.5, n.2, p. 1715, 2020.

31. OPAS. Saúde Digital: uma estratégia para manter a assistência à saúde de pessoas que vivem com doenças não transmissíveis durante a pandemia de COVID-19; In: Organização Panamericana da Saúde. Departamento de Evidência e Inteligência para Ação em Saúde. Disponível em: <https://pesquisa.bvsalud.org/porta I/resource/pt/biblio-1117108>. Acesso em: 25 nov. 2020.

32. SARBADHIKARI, S. and SARBADHIKARI, S.N.; The global experience of digital health interventions in COVID-19 management. Indian Journal of Public Health, v. 64 (Suplemento), p.S117-S124, 2020.

33. GLERIANO, J. S.; FABRO, G. C. R.; TOMAZ, W. B.; GOULART, B. F.; CHAVES, L. D. P. Reflexões sobre a gestão do Sistema Único de Saúde para a coordenação no enfrentamento da COVID-19. Escola Anna Nery, v. 24, 202 